\title{
DESIGNING PENALTY FUNCTIONS IN HIGH DIMENSIONAL PROBLEMS: THE ROLE OF TUNING PARAMETERS
}

\author{
Ting-Huei Chen, Wei Sun, and Jason P. Fine \\ Laval University, Fred Hutchinson Cancer Research Center, UNC Chapel Hill \\ Supplementary Material
}

\section{Supplementary Theoretical Results}

\subsection{Conditions for Weak Oracle Property}

The following proposition A1 is the same as the proposition 4 of Fan and Lv (2011), and it is needed for the following proof.

\section{Proposition A1.}

Let $y=\left(y_{1}, \ldots, y_{n}\right)^{\top}$ be the observed values of response variable. Let $a=\left(a_{1}, \ldots, a_{n}\right)^{\top} . P\left(\left|a^{\top} y-a^{\top} \mu\left(\theta_{0}\right)\right|>\right.$ $\left.\|a\|_{2} \varepsilon\right) \leq 2 e^{-\sigma \varepsilon^{2}}$, where $\varepsilon \in(0, \infty)$ and $\sigma=2 /(d-c)^{2}$ if $y$ are bounded in $[c, d]$; and $\varepsilon \in\left(0,\|a\|_{2} /\|a\|_{\infty}\right)$ and $\sigma=1 /\left(2 v_{0}+2 M\right)$, if $y$ are unbounded and there exist some $M \in(0, \infty)$ and $v_{0} \in(0, \infty)$ such that $\max _{i=1, \ldots, n} E\left[\exp \left\{\left|y_{i}-\mu\left(\theta_{0, i}\right)\right| / M\right\}-1-\left|y_{i}-\mu\left(\theta_{0, i}\right)\right| / M\right] M^{2} \leq v_{0} / 2$.

\section{Supplementary for computation algorithm}

We obtain the penalized estimates using SCAD or MCP by the coordinate descent algorithms implemented in the R package NCVREG (Breheny and Huang, 2011). We implement the penalized estimation using SICA and Log penalties by a combination of the coordinate descent algorithm and Local Linear Approximation (LLA) (Zou and Li, 2008). Specifically, we update the estimate of each regression coefficient sequentially (which is the coordinate decent part), and the solution of each coefficient is obtained after applying a local linear approximation:

$$
p_{\varpi}\left(\left|\beta_{j}\right|\right) \approx p_{\varpi}\left(\left|\hat{\beta}_{j}^{(k)}\right|\right)+p_{\varpi}^{\prime}\left(\left|\hat{\beta}_{j}^{(k)}\right|\right)\left(\left|\beta_{j}\right|-\left|\hat{\beta}_{j}^{(k)}\right|\right),
$$

where $\hat{\beta}_{j}^{(k)}$ is the estimate of regression coefficient $\beta_{j}$ at the $k$-th iteration.

We present the computational algorithms for linear and logistic regression separately. The objective function for linear regression is:

$$
Q_{n}(\beta)=-\frac{1}{2 n}(y-X \beta)^{\top}(y-X \beta)-\sum_{j=1}^{p} p_{\varpi}\left(\left|\beta_{j}\right|\right) .
$$

After applying LLA for the penalty function, the objective function to be maximized at the $(k+1)$-th step, while solving for $\beta_{j}$, is

$$
Q_{n}^{(k+1)}\left(\beta_{j}\right)=-\frac{1}{2 n}\left\|y-X_{-j} \hat{\beta}_{-j}^{(k)}-x_{j} \beta_{j}\right\|^{2}+\sum_{j=1}^{p} p_{\varpi}^{\prime}\left(\left|\hat{\beta}_{j}^{(k)}\right|\right)\left|\beta_{j}\right|,
$$


where $X_{-j}$ is the matrix $X$ without the $j$ th column, and $\hat{\beta}_{-j}^{(k)}$ is $\hat{\beta}^{(k)}$ without the $j$ th element. By letting $\partial Q_{n}^{(k+1)}\left(\beta_{j}\right) / \partial \beta_{j}=$ 0 , we can obtain the solution for $\beta_{j}$

$$
\left\{\begin{array}{ll}
\hat{\beta}_{j}^{(k+1)}=0 & \text { if }\left|z_{j}^{(k)}\right| \leq v_{j}^{-1} p_{\varpi}^{\prime}\left(\left|\hat{\beta}_{j}^{(k)}\right|\right) \\
\hat{\beta}_{j}^{(k+1)}=\operatorname{sgn}\left(\hat{\beta}_{j}^{(k)}\right)\left[\left|z_{j}^{(k)}\right|-v_{j}^{-1} p_{\varpi}^{\prime}\left(\left|\hat{\beta}_{j}^{(k)}\right|\right)\right] & \text { if }\left|z_{j}^{(k)}\right|>v_{j}^{-1} p_{\varpi}^{\prime}\left(\left|\hat{\beta}_{j}^{(k)}\right|\right)
\end{array},\right.
$$

where $z_{j}^{(k)}=x_{j}\left(y-X_{-j} \beta_{-j}^{(k)}\right) / v_{j}$, and $v_{j}=x_{j}^{\top} x_{j}$.

The penalized likelihood for logistic regression is

$$
Q_{n}(\beta)=\frac{1}{n} \sum_{i=1}^{n}\left\{y_{i} \log \frac{\pi_{i}}{1-\pi_{i}}+\log \left(1-\pi_{i}\right)\right\}-\sum_{j=1}^{p} p_{\varpi}\left(\left|\beta_{j}\right|\right),
$$

where $\pi_{i}=\operatorname{Pr}\left(y_{i}=1\right)$. By applying the iteratively reweighted least squares algorithm (McCullagh and Nelder, 1989) and the LLA of the penalty function, the objective function to be maximized at the $(k+1)$-th step, while solving for $\beta_{j}$, is

$$
\begin{aligned}
Q_{n}^{(k+1)}\left(\beta_{j}\right) \approx & -\frac{1}{2 n}\left(\tilde{y}^{(k)}-X_{-j} \hat{\beta}_{-j}^{(k)}-x_{j} \beta_{j}\right)^{\top} W^{(k)}\left(\tilde{y}^{(k)}-X_{-j} \hat{\beta}_{-j}^{(k)}-x_{j} \beta_{j}\right) \\
& +\sum_{j=1}^{p} p_{\varpi}^{\prime}\left(\left|\hat{\beta}_{j}^{(k)}\right|\right)\left|\beta_{j}\right|,
\end{aligned}
$$

where $\tilde{y}^{(k)}=X \hat{\beta}^{(k)}+\left(W^{(k)}\right)^{-1}\left(y-\pi^{(k)}\right), W^{(k)}$ is a diagonal matrix with the $i$-th diagonal element $w_{i}^{(k)}=$ $\pi_{i}^{(k)}\left(1-\pi_{i}^{(k)}\right)$, and $\pi_{i}^{(k)}=\exp \left(X \hat{\beta}^{(k)}\right) /\left[1+\exp \left(X \hat{\beta}^{(k)}\right)\right]$. Letting $\partial Q_{n}^{(k+1)}\left(\beta_{j}\right) / \partial \beta_{j}=0$, the estimate of $\beta_{j}$ is

$$
\begin{cases}\hat{\beta}_{j}^{(k+1)}=0 & \text { if }\left|z_{j}^{(k)}\right| \leq v_{j}^{-1} p_{\varpi}^{\prime}\left(\left|\hat{\beta}_{j}^{(k)}\right|\right) \\ \hat{\beta}_{j}^{(k+1)}=\operatorname{sgn}\left(\hat{\beta}_{j}^{(k)}\right)\left[\left|z_{j}^{(k)}\right|-v_{j}^{-1} p_{\varpi}^{\prime}\left(\left|\hat{\beta}_{j}^{(k)}\right|\right)\right] & \text { if }\left|z_{j}^{(k)}\right|>v_{j}^{-1} p_{\varpi}^{\prime}\left(\left|\hat{\beta}_{j}^{(k)}\right|\right)\end{cases}
$$

where $z_{j}^{(k)}=x_{j}^{\top} W^{(k)}\left(\tilde{y}^{(k)}-X_{-j} \beta_{-j}^{(k)}\right)$ and $v_{j}=x_{j}^{\top} W^{(k)} x_{j}$.

The iterative estimation process ends if the maximum difference of the estimates of $\beta$ between consecutive iterations is less than $10^{-5}$.

We follow a strategy similar to the ones in Breheny and Huang (2011) to obtain an initial set of tuning parameter combinations. For SCAD and MCP, the tuning parameter $a$ is given as a constant or a vector of legitimate values such as $a>2$ for SCAD and $a>1$ for MCP (the implementation of MCP in the R package NCVREG requires $a>1$ ). The $\lambda$ 's for SCAD and MCP are given as $N$ numbers equally spaced on a log scale, with the largest one corresponding to the largest marginal effect size and the smallest one being a fraction of the largest one. In our experience, the fraction is set as $1 / 10$ from the linear model, and $1 / 100$ for the logistic model.

For SICA and Log, the tuning parameter $\tau$ is set as a constant or a vector of legitimate values such as $\tau>0$. The theoretical results in previous sections suggest that $\tau$ should be much smaller than the minimum effect size. In practice, because we do not know which set of variables is important, we use the largest marginal effect size as the upper bound for $\tau$. Neither $\lambda$ nor $\tau$ alone determines the penalization strength. Instead, their combination in the form of the threshold $v_{j}^{-1} p_{\varpi}^{\prime}\left(\left|\hat{\beta}_{j}^{(k)}\right|\right)$ specifies the penalization strength. Without loss of generality, we assume $x_{j}(j=1, \ldots, p)$ is standardized with mean 0 and $v_{j}=\sum_{i=1}^{n} x_{i j}^{\top} x_{i j}=n$. It follows that the thresholds for SICA and the Log penalties are $p_{\varpi}^{\prime}(0) / v_{j}=p_{\varpi}^{\prime}(0) / n$. The largest threshold corresponds to the largest marginal coefficient estimates (by absolute value), denoted by $\hat{\beta}_{M}$, a predefined number of $\tau$ 's uniformly distributed on a $\log$ scale from 
$10^{-6}$ to $\hat{\beta}_{M}$, and the smallest threshold is $1 / 10$ of the largest one, i.e., $\hat{\beta}_{M} / 10$ for the linear model, and $1 / 100$ for the logistic model respectively:

$$
\left\{\text { Threshold }_{1}, \ldots, \text { Threshold } N\right\}=\left\{\hat{\beta}_{M}, \ldots, \frac{\hat{\beta}_{M}}{100}\right\} .
$$

For example, for Log penalty, the threshold in the first iteration is $\lambda /(n \tau)$. Then given a specific value of $\tau$ and a set of thresholds, $N \lambda$ 's can be generated based on the equation:

$$
\left\{\lambda_{1} / n \tau, \ldots, \lambda_{N} / n \tau\right\}=\left\{\text { Threshold }_{1}, \ldots, \text { Threshold } \mathrm{T}_{N}\right\}
$$

A similar strategy is used to determine the initial set of tuning parameters for SICA.

\section{Supplementary results for simulation analysis}

\section{Supplementary results for real data analysis}

Since the algorithm is based on coordinate decent, the results might be dependent on the order of updating the coefficients. Different updating orders within a chromosome did not have noticeable effect, and thus we carried out analysis with 40 different chromosome updating orders (including 39 random orders and one following the order of chromosome index) while the SNPs within each chromosome were ordered by their genomic positions. The final chosen SNPs were selected in more than 20 updating orders. 
Table 1: Simulation results for penalized linear regression with $(n=222, p=12,656)$. The headers indicate the tuning parameter selection criterion (Oracle or the extended BIC) and the numbers in parentheses are the number of important covariates. For each penalty, we present the median of the number of true discoveries, false discoveries (in parentheses), and average bias of the true discoveries (in brackets) across 100 simulations. - denotes missing value and it happens when the null model is chosen by the tuning parameter selection criterion.

\begin{tabular}{|c|c|c|c|}
\hline & \multicolumn{3}{|c|}{ Number of important variables } \\
\hline & 12 & 16 & 20 \\
\hline Selection criterion & \multicolumn{3}{|c|}{ Oracle $(w t=1)$} \\
\hline $\operatorname{SCAD}_{\lambda_{n}, a_{n}}$ & $11(23)[0.087]$ & $14(50)[0.07]$ & $16(73)[0.119]$ \\
\hline $\mathrm{MCP}_{\lambda_{n}, a_{n}}$ & $11(1)[0.077]$ & $14(4)[0.074]$ & $14(37.5)[0.155]$ \\
\hline $\log _{\lambda_{n}, \tau_{n}}$ & $11(2)[0.07]$ & $13.5(5)[0.088]$ & $13(32.5)[0.179]$ \\
\hline $\operatorname{SICA}_{\lambda_{n}, \tau_{n}}$ & $11(2)[0.069]$ & $14(4)[0.072]$ & $13(17)[0.166]$ \\
\hline $\mathrm{SCAD}_{\lambda_{n}}$ & $11(38.5)[0.114]$ & $14(67)[0.083]$ & $15(73)[0.145]$ \\
\hline $\mathrm{MCP}_{\lambda_{n}}^{{ }_{n}}$ & $11(3)[0.146]$ & $13(17)[0.105]$ & $10(17)[0.235]$ \\
\hline $\log _{\lambda_{n}}$ & $11(9.5)[0.232]$ & $13(76.5)[0.27]$ & $13(82.5)[0.287]$ \\
\hline SICA $_{\lambda_{n}}$ & $11(4)[0.207]$ & $13(47.5)[0.237]$ & $13(61)[0.246]$ \\
\hline Selection criterion & \multicolumn{3}{|c|}{$\operatorname{Oracle}(w t=5)$} \\
\hline $\operatorname{SCAD}_{\lambda_{n}, a_{n}}$ & $11(8)[0.259]$ & $14(39)[0.077]$ & $16(69)[0.129]$ \\
\hline $\mathrm{MCP}_{\lambda_{n}, a_{n}}$ & $11(1)[0.079]$ & $14(3)[0.073]$ & 14 (19) [0.149] \\
\hline $\log _{\lambda_{n}, \tau_{n}}$ & 11 (1) [0.069] & $13(5)[0.087]$ & $13(18)[0.134]$ \\
\hline $\mathrm{SICA}_{\lambda_{n}, \tau_{n}}$ & $11(1)[0.068]$ & $13(3)[0.072]$ & $12(12)[0.159]$ \\
\hline $\operatorname{SCAD}_{\lambda_{n}}$ & $11(9)[0.32]$ & $14(53.5)[0.118]$ & $15(65)[0.178]$ \\
\hline $\mathrm{MCP}_{\lambda_{n}}$ & $11(2)[0.188]$ & $13(11.5)[0.145]$ & $10(16)[0.248]$ \\
\hline $\log _{\lambda_{n}}$ & $11(3)[0.285]$ & $12(17)[0.34]$ & $12(52.5)[0.322]$ \\
\hline $\operatorname{SICA}_{\lambda_{n}}$ & $11(2)[0.241]$ & $12(12.5)[0.301]$ & $13(40.5)[0.267]$ \\
\hline Selection criterion & \multicolumn{3}{|c|}{ Oracle $(w t=10)$} \\
\hline $\mathrm{SCAD}_{\lambda_{n}, a_{n}}$ & $11(7)[0.307]$ & $14(36)[0.1]$ & $16(57.5)[0.149]$ \\
\hline $\mathrm{MCP}_{\lambda_{n}, a_{n}}$ & $11(1)[0.083]$ & 14 (3) [0.073] & $14(16.5)[0.162]$ \\
\hline $\log _{\lambda_{n}, \tau_{n}}$ & 11 (1) [0.069] & $13(4)[0.085]$ & $12(16)[0.138]$ \\
\hline $\operatorname{SICA}_{\lambda_{n}, \tau_{n}}$ & 11 (1) [0.068] & 13 (3) [0.069] & $11(10)[0.15]$ \\
\hline $\mathrm{SCAD}_{\lambda_{n}}$ & $11(7)[0.332]$ & $14(49.5)[0.161]$ & $15(61)[0.213]$ \\
\hline $\mathrm{MCP}_{\lambda_{n}}$ & $11(2)[0.212]$ & $13(11)[0.164]$ & $10(15)[0.262]$ \\
\hline $\log _{\lambda_{n}}$ & $10(3)[0.291]$ & $10(6.5)[0.37]$ & 7 (11) $[0.352]$ \\
\hline SICA $_{\lambda_{n}}$ & $10(2)[0.249]$ & $12(8.5)$ [0.319] & $11(16.5)$ [0.29] \\
\hline Selection criterion & \multicolumn{3}{|c|}{ Oracle $(w t=15)$} \\
\hline $\operatorname{SCAD}_{\lambda_{n}, a_{n}}$ & $11(7)[0.317]$ & $14(33)[0.111]$ & $16(52)[0.178]$ \\
\hline $\mathrm{MCP}_{\lambda_{n}, a_{n}}$ & 11 (1) [0.088] & 14 (3) $[0.076]$ & $13(13.5)[0.168]$ \\
\hline $\log _{\lambda_{n}, \tau_{n}}$ & 11 (1) [0.069] & $13(4)[0.084]$ & $12(13.5)[0.138]$ \\
\hline $\operatorname{SICA}_{\lambda_{n}, \tau_{n}}$ & $11(1)[0.068]$ & 13 (3) [0.069] & $11(10)[0.148]$ \\
\hline $\operatorname{SCAD}_{\lambda_{n}}$ & $11(7)[0.338]$ & $14(45.5)[0.21]$ & 15 (55.5) [0.247] \\
\hline $\mathrm{MCP}_{\lambda_{n}}$ & $11(2)[0.214]$ & 13 (10) [0.173] & $10(15)[0.271]$ \\
\hline $\log _{\lambda_{n}}$ & $10(3)[0.294]$ & $9(4.5)[0.379]$ & $3.5(0.5)[0.364]$ \\
\hline $\operatorname{SICA}_{\lambda_{n}}$ & $10(2)[0.251]$ & $11.5(8)[0.324]$ & $9(11)[0.295]$ \\
\hline Selection criterion & \multicolumn{3}{|c|}{ Ext BIC } \\
\hline $\operatorname{SCAD}_{\lambda_{n}, a_{n}}$ & $-(-)[-]$ & $-(-)[-]$ & $-(-)[-]$ \\
\hline $\mathrm{MCP}_{\lambda_{n}, a_{n}}$ & $10(32)[0.081]$ & 13 (39) [0.089] & $7(45)[0.113]$ \\
\hline $\log _{\lambda_{n}, \tau_{n}}$ & $11(2)[0.064]$ & $13(5)[0.084]$ & 11 (17) [0.113] \\
\hline $\mathrm{SICA}_{\lambda_{n}, \tau_{n}}$ & $10(3)[0.061]$ & $13(6)[0.067]$ & 10.5 (12) [0.1] \\
\hline $\operatorname{SCAD}_{\lambda_{n}}$ & $-(-)[-]$ & $-(-)[-]$ & $-(-)[-]$ \\
\hline $\mathrm{MCP}_{\lambda_{n}}$ & $10(3)[0.147]$ & $-(-)[-]$ & $-(-)[-]$ \\
\hline $\log _{\lambda_{n}}$ & $1(0.5)[0.291]$ & $1(0)[0.456]$ & 1 (1) [0.437] \\
\hline SICA $_{\lambda_{n}}$ & $10(2)[0.215]$ & $1(0)[0.421]$ & $1(1)[0.329]$ \\
\hline
\end{tabular}


Table 2: Simulation results for penalized linear regression with $(n=222, p=4000)$. The headers indicate the tuning parameter selection criterion and the numbers in parentheses are the number of important covariates. For each penalty, we present the median of the number of true discoveries, false discoveries (in parentheses), and average bias of the true discoveries (in brackets) across 100 simulations. - denotes missing value and it happens when the null model is chosen by the tuning parameter selection criterion.

\begin{tabular}{|c|c|c|c|}
\hline & \multicolumn{3}{|c|}{ Number of important variables } \\
\hline & 12 & 16 & 20 \\
\hline Selection criterion & \multicolumn{3}{|c|}{ Oracle $(w t=1)$} \\
\hline $\operatorname{SCAD}_{\lambda_{n}, a_{n}}$ & $11(7)[0.076]$ & $15(20.5)[0.064]$ & $19(23.5)[0.065]$ \\
\hline $\mathrm{MCP}_{\lambda_{n}, a_{n}}$ & $11(1)[0.065]$ & $15(2)[0.063]$ & $18(3)[0.072]$ \\
\hline $\log _{\lambda_{n}, \tau_{n}}$ & $11(1)[0.065]$ & 15 (3) [0.073] & $18(5)[0.082]$ \\
\hline $\operatorname{SICA}_{\lambda_{n}, \tau_{n}}$ & $11(1)[0.066]$ & $15(2)[0.061]$ & $18(5)[0.076]$ \\
\hline $\operatorname{SCAD}_{\lambda_{n}}$ & $11(11.5)[0.146]$ & 15 (51.5) [0.07] & $19(50)[0.077]$ \\
\hline $\mathrm{MCP}_{\lambda_{n}}$ & $10(2)[0.134]$ & 14 (10.5) [0.099] & 18 (12) [0.099] \\
\hline $\log _{\lambda_{n}}$ & $10.5(4)[0.22]$ & $14(61)[0.232]$ & $19(70)[0.206]$ \\
\hline SICA $_{\lambda_{n}}$ & $10(3)[0.189]$ & $14(35.5)[0.208]$ & 19 (39) [0.181] \\
\hline Selection criterion & \multicolumn{3}{|c|}{ Oracle $(w t=5)$} \\
\hline $\mathrm{SCAD}_{\lambda_{n}, a_{n}}$ & $11(3)[0.242]$ & $15(15)[0.071]$ & 19.5 (18) [0.08] \\
\hline $\mathrm{MCP}_{\lambda_{n}, a_{n}}$ & $11(1)[0.064]$ & $15(2)[0.064]$ & $18(2)[0.071]$ \\
\hline $\log _{\lambda_{n}, \tau_{n}}$ & $11(1)[0.065]$ & $15(3)[0.071]$ & $18(5)[0.079]$ \\
\hline $\operatorname{SICA}_{\lambda_{n}, \tau_{n}}$ & $11(1)[0.066]$ & $15(2)[0.061]$ & $18(3)[0.069]$ \\
\hline $\mathrm{SCAD}_{\lambda_{n}}$ & $11(3)[0.294]$ & 15 (36.5) [0.102] & $19(36.5)[0.11]$ \\
\hline $\mathrm{MCP}_{\lambda_{n}}$ & $10(2)[0.167]$ & $14(7)[0.136]$ & $18(8)[0.135]$ \\
\hline $\log _{\lambda_{n}}$ & $10(2)$ [0.267] & $14(14.5)[0.321]$ & $19(32)[0.254]$ \\
\hline $\mathrm{SICA}_{\lambda_{n}}$ & $10(2)[0.224]$ & $14(10)[0.284]$ & $18(17.5)[0.226]$ \\
\hline Selection criterion & \multicolumn{3}{|c|}{ Oracle $(w t=10)$} \\
\hline $\mathrm{SCAD}_{\lambda_{n}, a_{n}}$ & $11(2)[0.276]$ & 15 (14) [0.074] & 19 (17) [0.089] \\
\hline $\mathrm{MCP}_{\lambda_{n}, a_{n}}$ & 11 (1) $[0.065]$ & 15 (1.5) [0.064] & $18(2)[0.071]$ \\
\hline $\log _{\lambda_{n}, \tau_{n}}$ & $11(1)[0.065]$ & 15 (3) [0.072] & $18(4)$ [0.077] \\
\hline $\operatorname{SICA}_{\lambda_{n}, \tau_{n}}$ & $11(1)$ [0.066] & $15(2)[0.061]$ & $18(3)[0.069]$ \\
\hline $\operatorname{SCAD}_{\lambda_{n}}$ & $11(2)[0.314]$ & $15(30)[0.136]$ & $19(35)[0.12]$ \\
\hline $\mathrm{MCP}_{\lambda_{n}}$ & $10(1)[0.174]$ & $14(6)[0.156]$ & $18(6)[0.157]$ \\
\hline $\log _{\lambda_{n}}$ & $10(2)[0.278]$ & $12(7)[0.348]$ & $18(23.5)[0.276]$ \\
\hline $\operatorname{SICA}_{\lambda_{n}}$ & $10(1.5)[0.225]$ & $13(8)[0.303]$ & $18(14)[0.237]$ \\
\hline Selection criterion & \multicolumn{3}{|c|}{ Oracle $(w t=15)$} \\
\hline $\operatorname{SCAD}_{\lambda_{n}, a_{n}}$ & $11(2)[0.286]$ & 15 (13) [0.084] & $19(15.5)[0.094]$ \\
\hline $\mathrm{MCP}_{\lambda_{n}, a_{n}}$ & $11(1)[0.065]$ & 15 (1.5) [0.064] & $18(2)[0.071]$ \\
\hline $\log _{\lambda_{n}, \tau_{n}}$ & $11(1)[0.066]$ & 15 (3) [0.072] & $18(4)[0.077]$ \\
\hline $\operatorname{SICA}_{\lambda_{n}, \tau_{n}}$ & 11 (1) [0.066] & $15(2)[0.061]$ & $18(3)[0.069]$ \\
\hline $\operatorname{SCAD}_{\lambda_{n}}$ & $11(2)[0.318]$ & $14(22.5)[0.178]$ & $19(33.5)[0.131]$ \\
\hline $\mathrm{MCP}_{\lambda_{n}}$ & $10(1)[0.174]$ & $14(6)[0.163]$ & $18(6)$ [0.159] \\
\hline $\log _{\lambda_{n}}$ & $10(2)[0.279]$ & $12(7)[0.351]$ & $18(22)[0.284]$ \\
\hline $\operatorname{SICA}_{\lambda_{n}}$ & $10(1)[0.226]$ & $13(7.5)[0.307]$ & $18(12)[0.247]$ \\
\hline Selection criterion & \multicolumn{3}{|c|}{ Ext BIC } \\
\hline $\operatorname{SCAD}_{\lambda_{n}, a_{n}}$ & $11(5)[0.1]$ & $0(0)[0.061]$ & $0(0)[0.063]$ \\
\hline $\mathrm{MCP}_{\lambda_{n}, a_{n}}$ & $10.5(31)[0.072]$ & $14(39)$ [0.087] & $17.5(36)[0.092]$ \\
\hline $\log _{\lambda_{n}, \tau_{n}}$ & $11(2)$ [0.059] & $15(4)[0.075]$ & 18 (12) [0.087] \\
\hline $\operatorname{SICA}_{\lambda_{n}, \tau_{n}}$ & $11(3)[0.06]$ & $14(4)[0.065]$ & $18(27)[0.082]$ \\
\hline $\mathrm{SCAD}_{\lambda_{n}}$ & $10(3.5)$ [0.187] & $0(0)[0.081]$ & $0(0)[0.079]$ \\
\hline $\mathrm{MCP}_{\lambda_{n}}$ & $10(3)$ [0.119] & $14(9)[0.093]$ & 18 (10) [0.098] \\
\hline $\log _{\lambda_{n}}$ & $10(3)[0.225]$ & $1(0)[0.452]$ & $1(0)[0.423]$ \\
\hline $\operatorname{SICA}_{\lambda_{n}}$ & $10(3)[0.181]$ & $1(0)[0.369]$ & $1(0)[0.329]$ \\
\hline
\end{tabular}


Table 3: Simulation results for penalized logistic regression $(n=750, p=10,156)$. The headers indicate the tuning parameter selection criterion and the numbers in parentheses are the number of important covariates. For each penalty, we present the median of the number of true discoveries, the number of false discoveries (in parentheses), and the average bias of true discoveries (in brackets) across 100 simulations. - denotes missing value and it happens when the null model is chosen by the tuning parameter selection criterion.

\begin{tabular}{|c|c|c|c|}
\hline & 4 & 8 & 12 \\
\hline Selection criterion & \multicolumn{3}{|c|}{ Oracle $(w t=1)$} \\
\hline $\mathrm{SCAD}_{\lambda_{n}, a_{n}}$ & $4(0)[0.373]$ & $8(45.5)[0.229]$ & $12(77)[0.222]$ \\
\hline $\mathrm{MCP}_{\lambda_{n}, a_{n}}$ & $4(0)[0.094]$ & 7 (1) [0.178] & 12 (13) [0.179] \\
\hline $\log _{\lambda_{n}, \tau_{n}}$ & $4(0)[0.083]$ & $8(0)[0.087]$ & $11(1)[0.1]$ \\
\hline $\operatorname{SICA}_{\lambda_{n}, \tau_{n}}$ & $4(0)[0.088]$ & 7 (0) [0.09] & $11(0)[0.098]$ \\
\hline $\mathrm{SCAD}_{\lambda_{n}}$ & $4(0)[0.476]$ & $8(86)[0.286]$ & $12(82)$ [0.409] \\
\hline $\mathrm{MCP}_{\lambda_{n}}$ & $4(0)[0.341]$ & $8(22.5)[0.258]$ & $12(53.5)[0.211]$ \\
\hline $\log _{\lambda_{n}}$ & $4(0)[0.436]$ & $8(82)[0.391]$ & $12(82)[0.433]$ \\
\hline $\operatorname{SICA}_{\lambda_{n}}$ & $4(0)[0.38]$ & $8(18.5)[0.381]$ & $12(81)[0.374]$ \\
\hline Selection criterion & \multicolumn{3}{|c|}{ Oracle $(w t=5)$} \\
\hline $\operatorname{SCAD}_{\lambda_{n}, a_{n}}$ & $4(0)[0.478]$ & $7(0)[0.529]$ & $12(27)[0.367]$ \\
\hline $\mathrm{MCP}_{\lambda_{n}, a_{n}}$ & $4(0)[0.094]$ & $7(0)[0.22]$ & $11(2)[0.292]$ \\
\hline $\log _{\lambda_{n}, \tau_{n}}$ & $4(0)[0.083]$ & $7(0)$ [0.084] & $11(0)$ [0.097] \\
\hline $\mathrm{SICA}_{\lambda_{n}, \tau_{n}}$ & $4(0)[0.088]$ & $7(0)$ [0.09] & $11(0)$ [0.099] \\
\hline $\operatorname{SCAD}_{\lambda_{n}}$ & $4(0)[0.49]$ & $7(0)[0.543]$ & $11(3)[0.582]$ \\
\hline $\mathrm{MCP}_{\lambda_{n}}$ & $4(0)[0.368]$ & $7(0)[0.43]$ & 11 (3) [0.49] \\
\hline $\log _{\lambda_{n}}$ & $4(0)[0.446]$ & $7(0)[0.512]$ & $11(2.5)[0.559]$ \\
\hline $\operatorname{SICA}_{\lambda_{n}}$ & $4(0)[0.384]$ & $7(0)[0.468]$ & $11(2)[0.526]$ \\
\hline Selection criterion & \multicolumn{3}{|c|}{ Oracle $(w t=10)$} \\
\hline $\operatorname{SCAD}_{\lambda_{n}, a_{n}}$ & $4(0)[0.478]$ & $7(0)[0.532]$ & $11(2)[0.575]$ \\
\hline $\mathrm{MCP}_{\lambda_{n}, a_{n}}$ & $4(0)[0.094]$ & $7(0)[0.233]$ & $11(1)[0.334]$ \\
\hline $\log _{\lambda_{n}, \tau_{n}}$ & $4(0)[0.083]$ & $7(0)[0.084]$ & $11(0)[0.097]$ \\
\hline $\operatorname{SICA}_{\lambda_{n}, \tau_{n}}$ & $4(0)[0.088]$ & 7 (0) [0.09] & $11(0)[0.099]$ \\
\hline $\operatorname{SCAD}_{\lambda_{n}}$ & $4(0)[0.49]$ & $7(0)[0.543]$ & $11(2)[0.588]$ \\
\hline $\mathrm{MCP}_{\lambda_{n}}$ & $4(0)[0.368]$ & $7(0)[0.436]$ & $11(2)[0.512]$ \\
\hline $\log _{\lambda_{n}}$ & $4(0)[0.446]$ & $7(0)[0.512]$ & $11(1)[0.567]$ \\
\hline SICA $_{\lambda_{n}}$ & $4(0)[0.384]$ & $7(0)[0.471]$ & $11(2)[0.537]$ \\
\hline Selection criterion & \multicolumn{3}{|c|}{ Oracle $(w t=15)$} \\
\hline $\mathrm{SCAD}_{\lambda_{n}, a_{n}}$ & $4(0)[0.478]$ & $7(0)[0.532]$ & $11(2)[0.581]$ \\
\hline $\mathrm{MCP}_{\lambda_{n}, a_{n}}$ & $4(0)[0.094]$ & $7(0)[0.247]$ & $11(1)[0.376]$ \\
\hline $\log _{\lambda_{n}, \tau_{n}}$ & $4(0)[0.083]$ & $7(0)[0.084]$ & $11(0)[0.097]$ \\
\hline $\mathrm{SICA}_{\lambda_{n}, \tau_{n}}$ & $4(0)[0.088]$ & $7(0)[0.09]$ & $11(0)[0.099]$ \\
\hline $\operatorname{SCAD}_{\lambda_{n}}$ & $4(0)[0.49]$ & $7(0)[0.543]$ & $11(2)[0.589]$ \\
\hline $\mathrm{MCP}_{\lambda_{n}}$ & $4(0)[0.368]$ & $7(0)[0.435]$ & $11(2)[0.514]$ \\
\hline $\log _{\lambda_{n}}$ & $4(0)[0.446]$ & $7(0)[0.512]$ & 11 (1) [0.569] \\
\hline $\operatorname{SICA}_{\lambda_{n}}$ & $4(0)[0.384]$ & $7(0)[0.471]$ & $11(1)[0.541]$ \\
\hline Selection criterion & \multicolumn{3}{|c|}{ Ext BIC } \\
\hline $\operatorname{SCAD}_{\lambda_{n}, a_{n}}$ & $4(0)[0.395]$ & $6(0)[0.432]$ & $-(-)[-]$ \\
\hline $\mathrm{MCP}_{\lambda_{n}, a_{n}}$ & $4(0)[0.097]$ & $6(2)[0.143]$ & $11(6)[0.225]$ \\
\hline $\log _{\lambda_{n}, \tau_{n}}$ & $4(0)[0.091]$ & 7 (1) $[0.11]$ & 11 (1) [0.107] \\
\hline $\operatorname{SICA}_{\lambda_{n}, \tau_{n}}$ & $4(0)[0.091]$ & 7 (1) $[0.11]$ & $12(1)[0.11]$ \\
\hline $\operatorname{SCAD}_{\lambda_{n}}$ & $4(0)[0.463]$ & $5(0)[0.518]$ & $-(-)[-]$ \\
\hline $\mathrm{MCP}_{\lambda_{n}}$ & $4(0)[0.325]$ & $7(0)$ [0.404] & $6(0)[0.516]$ \\
\hline $\log _{\lambda_{n}}$ & $4(0)[0.421]$ & $6(0)$ [0.499] & $1(0)$ [0.554] \\
\hline $\operatorname{SICA}_{\lambda_{n}}$ & $4(0)[0.366]$ & $6(0)[0.451]$ & $2(0)[0.517]$ \\
\hline
\end{tabular}




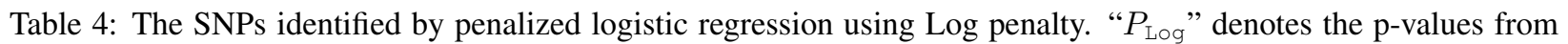
the model selected using Log penalty, which includes age, gender, 23 PCs, and 38 SNPs. " $P_{\text {marginal" denotes the }}$ p-values by univariate SNP logistic regression controlled by age, gender and 23 PCs.

$\begin{array}{llrll}\text { SNP ID } & \text { Chromosome } & \text { Position } & P_{\text {Log }} & P_{\text {marginal }} \\ \text { rs11580545 } & 1 & 58838246 & 1.3 \mathrm{E}-05 & 1.8 \mathrm{E}-04 \\ \text { rs6672282 } & 1 & 80199565 & 4.1 \mathrm{E}-07 & 5.3 \mathrm{E}-05 \\ \text { rs1413234 } & 1 & 97685992 & 6.9 \mathrm{E}-06 & 6.4 \mathrm{E}-05 \\ \text { rs10802409 } & 1 & 246632031 & 1 \mathrm{E}-05 & 9.3 \mathrm{E}-05 \\ \text { rs1155337 } & 2 & 13770677 & 1 \mathrm{E}-06 & 2.7 \mathrm{E}-04 \\ \text { rs11892969 } & 2 & 18973451 & 1.6 \mathrm{E}-07 & 1.1 \mathrm{E}-04 \\ \text { rs1509360 } & 2 & 22616777 & 1.2 \mathrm{E}-06 & 3.1 \mathrm{E}-06 \\ \text { rs7601770 } & 2 & 62658256 & 2.8 \mathrm{E}-05 & 5.4 \mathrm{E}-06 \\ \text { rs3731684 } & 2 & 108618514 & 5.8 \mathrm{E}-06 & 2.8 \mathrm{E}-05 \\ \text { rs709937 } & 2 & 233744021 & 3.5 \mathrm{E}-07 & 4.2 \mathrm{E}-05 \\ \text { rs9825041 } & 3 & 45475993 & 5.9 \mathrm{E}-06 & 1.5 \mathrm{E}-05 \\ \text { rs9876866 } & 3 & 84836382 & 8.5 \mathrm{E}-05 & 6.2 \mathrm{E}-05 \\ \text { rs11926785 } & 3 & 106089518 & 1.9 \mathrm{E}-05 & 1.9 \mathrm{E}-05 \\ \text { rs3843375 } & 3 & 196813908 & 9.7 \mathrm{E}-06 & 2.7 \mathrm{E}-04 \\ \text { rs11732323 } & 4 & 55643116 & 1.4 \mathrm{E}-05 & 4.4 \mathrm{E}-05 \\ \text { rs17021364 } & 4 & 95047893 & 4.1 \mathrm{E}-05 & 2.6 \mathrm{E}-06 \\ \text { rs12644237 } & 4 & 190284268 & 3.2 \mathrm{E}-05 & 4.3 \mathrm{E}-06 \\ \text { rs371256 } & 5 & 73667222 & 3.6 \mathrm{E}-06 & 3.6 \mathrm{E}-05 \\ \text { rs25820 } & 5 & 128991232 & 4 \mathrm{E}-06 & 5.3 \mathrm{E}-05 \\ \text { rs4835789 } & 5 & 137739040 & 5.7 \mathrm{E}-06 & 6.5 \mathrm{E}-06 \\ \text { rs4713419 } & 6 & 30993236 & 1.2 \mathrm{E}-06 & 2.5 \mathrm{E}-05 \\ \text { rs4337917 } & 6 & 167111068 & 1.6 \mathrm{E}-06 & 7.3 \mathrm{E}-06 \\ \text { rs1568777 } & 7 & 21681379 & 7.2 \mathrm{E}-05 & 3.5 \mathrm{E}-04 \\ \text { rs16921167 } & 8 & 56036859 & 3.7 \mathrm{E}-05 & 4.2 \mathrm{E}-06 \\ \text { rs6998613 } & 8 & 66074310 & 1.6 \mathrm{E}-06 & 3.1 \mathrm{E}-06 \\ \text { rs4743626 } & 9 & 105904199 & 3.4 \mathrm{E}-07 & 2.6 \mathrm{E}-06 \\ \text { rs11038064 } & 11 & 44591432 & 6.7 \mathrm{E}-05 & 1.8 \mathrm{E}-05 \\ \text { rs11061935 } & 12 & 1813774 & 3.5 \mathrm{E}-06 & 1.2 \mathrm{E}-05 \\ \text { rs16921193 } & 12 & 33798136 & 8.9 \mathrm{E}-07 & 1.5 \mathrm{E}-05 \\ \text { rs7337857 } & 13 & 53413435 & 1.2 \mathrm{E}-06 & 5 \mathrm{E}-05 \\ \text { rs2401751 } & 14 & 88946622 & 6.2 \mathrm{E}-05 & 1.9 \mathrm{E}-05 \\ \text { rs11642912 } & 16 & 77063426 & 3.8 \mathrm{E}-05 & 3 \mathrm{E}-05 \\ \text { rs9947138 } & 18 & 76157317 & 6.5 \mathrm{E}-07 & 3.9 \mathrm{E}-07 \\ \text { rs877567 } & 19 & 33782911 & 1.2 \mathrm{E}-05 & 1.5 \mathrm{E}-05 \\ \text { rs910389 } & 20 & 894722 & 4.6 \mathrm{E}-06 & 5.8 \mathrm{E}-05 \\ \text { rs6045692 } & 20 & 18901412 & 3.8 \mathrm{E}-06 & 5.4 \mathrm{E}-05 \\ \text { rs9625334 } & 22 & 21371227 & 2.2 \mathrm{E}-06 & 4.5 \mathrm{E}-06 \\ \text { rs9613228 } & 22 & 27002223 & 4.9 \mathrm{E}-05 & 1.4 \mathrm{E}-05\end{array}$


Table 5: The SNPs identified by penalized logistic regression using SICA penalty. " $P_{\text {SICA }}$ " denotes the p-values from the model selected using SICA penalty, which includes age, gender, 23 PCs, and 22 SNPs. "P $P_{\text {marginal" denotes the }}$ p-values by univariate SNP logistic regression controlled by age, gender and 23 PCs.

$\begin{array}{llrll}\text { SNP ID } & \text { Chromosome } & \text { Position } & P_{\text {SICA }} & P_{\text {marginal }} \\ \text { rs6672282 } & 1 & 80199565 & 7.1 \mathrm{E}-06 & 5.3 \mathrm{E}-05 \\ \text { rs1413234 } & 1 & 97685992 & 4.6 \mathrm{E}-06 & 6.4 \mathrm{E}-05 \\ \text { rs11892969 } & 2 & 18973451 & 4.7 \mathrm{E}-06 & 1.1 \mathrm{E}-04 \\ \text { rs1509360 } & 2 & 22616777 & 1.7 \mathrm{E}-06 & 3.1 \mathrm{E}-06 \\ \text { rs7601770 } & 2 & 62658256 & 3.8 \mathrm{E}-06 & 5.4 \mathrm{E}-06 \\ \text { rs3731684 } & 2 & 108618514 & 2.2 \mathrm{E}-05 & 2.8 \mathrm{E}-05 \\ \text { rs709937 } & 2 & 233744021 & 2 \mathrm{E}-06 & 4.2 \mathrm{E}-05 \\ \text { rs9825041 } & 3 & 45475993 & 4.5 \mathrm{E}-06 & 1.5 \mathrm{E}-05 \\ \text { rs11926785 } & 3 & 106089518 & 8.1 \mathrm{E}-06 & 1.9 \mathrm{E}-05 \\ \text { rs10030713 } & 4 & 95238536 & 5.4 \mathrm{E}-06 & 1.8 \mathrm{E}-06 \\ \text { rs12644237 } & 4 & 190284268 & 3.5 \mathrm{E}-05 & 4.3 \mathrm{E}-06 \\ \text { rs4835789 } & 5 & 137739040 & 3.6 \mathrm{E}-05 & 6.5 \mathrm{E}-06 \\ \text { rs4337917 } & 6 & 167111068 & 2.8 \mathrm{E}-06 & 7.3 \mathrm{E}-06 \\ \text { rs16921167 } & 8 & 56036859 & 1.7 \mathrm{E}-04 & 4.2 \mathrm{E}-06 \\ \text { rs6998613 } & 8 & 66074310 & 2.5 \mathrm{E}-06 & 3.1 \mathrm{E}-06 \\ \text { rs4743626 } & 9 & 105904199 & 1.7 \mathrm{E}-06 & 2.6 \mathrm{E}-06 \\ \text { rs11061935 } & 12 & 1813774 & 6.3 \mathrm{E}-06 & 1.2 \mathrm{E}-05 \\ \text { rs16921193 } & 12 & 33798136 & 5.5 \mathrm{E}-06 & 1.5 \mathrm{E}-05 \\ \text { rs9947138 } & 18 & 76157317 & 4.2 \mathrm{E}-07 & 3.9 \mathrm{E}-07 \\ \text { rs877567 } & 19 & 33782911 & 1.5 \mathrm{E}-05 & 1.5 \mathrm{E}-05 \\ \text { rs9625334 } & 22 & 21371227 & 3.8 \mathrm{E}-06 & 4.5 \mathrm{E}-06 \\ \text { rs9613228 } & 22 & 27002223 & 2.5 \mathrm{E}-05 & 1.4 \mathrm{E}-05\end{array}$




\section{Proofs}

\section{Proof of Theorem 2}

The following proof is similar to the proof of Theorem 2 of Fan and Lv (2011); we provide the proof for the generalized conditions for penalty functions.

For easier reference to the equations in Theorem 1, we present Theorem 1 here.

Theorem 1. (Characterization of PMLE): $\hat{\beta} \in R^{p}$ is a strict local maximizer of the non-concave penalized likelihood $\overline{Q_{n}(\beta)=l_{n}}(\beta)-\sum_{j=1}^{p} p_{\varpi}\left(\left|\beta_{j}\right|\right)$ if

$$
\begin{aligned}
X_{1}^{\top} \mu(\hat{\theta})-X_{1}^{\top} y+n p_{\varpi}^{\prime}\left(\hat{\beta}_{01}\right) & =0 \\
\left\|X_{2}^{\top}(y-\mu(\hat{\theta}))\right\|_{\infty}-n p_{\varpi}^{\prime}(0+) & <0 \\
\lambda_{\min }\left(X_{1}^{\top} \Sigma(\hat{\theta}) X_{1}\right)-n \kappa\left(p_{\varpi}, \hat{\beta}_{01}\right) & >0 .
\end{aligned}
$$

Let $\boldsymbol{\xi}=\left(\xi_{1}, \ldots, \xi_{p}\right)^{\top}=X^{\top}\left(y-\mu\left(X^{\top} \beta_{0}\right)\right)=X^{\top} \boldsymbol{\epsilon}$, and consider events

$$
\mathcal{E}_{1}=\left\{\left\|\boldsymbol{\xi}_{1}\right\|_{\infty} \leq \sigma^{-1 / 2} n^{1 / 2} \sqrt{\log n}\right\} \text { and } \mathcal{E}_{2}=\left\{\left\|\boldsymbol{\xi}_{2}\right\|_{\infty} \leq \sigma^{-1 / 2} n^{1 / 2} \sqrt{n^{\alpha} \log n}\right\}
$$

where $\boldsymbol{\xi}_{1}=\left(\xi_{1}, \ldots, \xi_{s}\right)^{\top}, \boldsymbol{\xi}_{2}=\left(\xi_{s+1}, \ldots, \xi_{p}\right)^{\top}$, and $\sigma=2 /(d-c)^{2}$ if $y$ are bounded or $\sigma=1 /\left(2 v_{0}+2 M\right)$ if $y$ are unbounded by the proposition A1. Under the event $\mathcal{E}_{1} \cap \mathcal{E}_{2}$, it can be shown that there exists a solution $\hat{\beta} \in \mathbf{R}^{p}$ to equations (1)-(3) with $\operatorname{sgn}(\hat{\beta})=\operatorname{sgn}\left(\beta_{0}\right)$ and $\left\|\hat{\beta}_{1}-\beta_{01}\right\|_{\infty}=o\left(n^{-\gamma_{0}} \sqrt{\log n}\right)$.

By applying proposition A1, and let $a=x_{j}$ for $j=1, \ldots, p$, and thus $\|a\|_{2}=\left\|x_{j}\right\|_{2}=\sqrt{n}$. For $\mathcal{E}_{1}$, let $\varepsilon=\sigma^{-1 / 2} \sqrt{\log n}$,

$$
P\left(\left|a^{\top} y-a^{\top} \mu\left(\theta_{0}\right)\right|>\|a\|_{2} \varepsilon\right) \leq 2 e^{-\sigma \varepsilon^{2}} \Rightarrow P\left(\left|\xi_{j}\right|>\sigma^{-1 / 2} n^{1 / 2} \sqrt{\log n}\right)<2 n^{-1} .
$$

In addition, for $\mathcal{E}_{2}$, let $\varepsilon=\sigma^{-1 / 2} \sqrt{n^{\alpha} \log n}$,

$$
P\left(\left|a^{\top} y-a^{\top} \mu\left(\theta_{0}\right)\right|>\|a\|_{2} \varepsilon\right) \leq 2 e^{-\sigma \varepsilon^{2}} \Rightarrow P\left(\left|\xi_{j}\right|>\sigma^{-1 / 2} n^{1 / 2} \sqrt{n^{\alpha} \log n}\right)<2 e^{-\left(n^{\alpha} \log n\right)} .
$$

Next, by Bonferroni's inequality

$$
\begin{aligned}
P\left(\mathcal{E}_{1} \cap \mathcal{E}_{2}\right) & \geq 1-\sum_{j=1}^{s} P\left(\left|\xi_{j}\right|>\sigma^{-1 / 2} n^{1 / 2} \sqrt{\log n}\right)-\sum_{j=s+1}^{p} P\left(\left|\xi_{j}\right|>\sigma^{-1 / 2} n^{1 / 2} \sqrt{n^{\alpha} \log n}\right) \\
& \geq 1-2\left[s n^{-1}+(p-s) e^{-\left(n^{\alpha} \log n\right)}\right] .
\end{aligned}
$$

Recall that $\beta_{0}=\left(\beta_{01}^{\top}, \beta_{02}^{\top}\right)^{\top} \in \mathbf{R}^{p}$ are the true coefficients, where $\beta_{01} \in \mathbf{R}^{s}, \beta_{02}=\mathbf{0} \in \mathbf{R}^{p-s}$. Let $\hat{\beta}=\left(\hat{\beta}_{1}^{\top}, \hat{\beta}_{2}^{\top}\right)^{\top} \in \mathbf{R}^{p}$ be the estimated coefficients. We will prove, under the event $\mathcal{E}_{1} \cap \mathcal{E}_{2}$, there is a penalized likelihood estimate such that $\hat{\beta}_{2}=\mathbf{0}$, and $\hat{\beta}_{1} \rightarrow \beta_{01}$ by $L_{\infty}$ norm.

$\underline{\text { Step 1: Consistency in the } s \text {-dimensional subspace }}$ 
For sufficiently large $n$, we will prove the existence of a solution $\hat{\beta}_{1} \in \mathbf{R}^{s}$ for the equation (1) of $X_{1}^{\top} \mu\left(X_{1} \hat{\beta}_{1}\right)-$ $X_{1}^{\top} y+n p_{\varpi}^{\prime}\left(\hat{\beta}_{1}\right)=0$ inside the hypercube

$$
\mathcal{N}=\left\{\boldsymbol{\delta} \in \mathbf{R}^{s}:\left\|\boldsymbol{\delta}-\beta_{01}\right\|_{\infty}=d_{n}=O\left(n^{-\gamma_{0}} \sqrt{\log n}\right)\right\}
$$

Define

$$
\begin{aligned}
\varphi(\boldsymbol{\delta}) & =X_{1}^{\top} \mu\left(X_{1} \boldsymbol{\delta}\right)-X_{1}^{\top} y+n p_{\varpi}^{\prime}(\boldsymbol{\delta}) \\
& =X_{1}^{\top} \mu\left(X_{1} \boldsymbol{\delta}\right)-X_{1}^{\top} \mu\left(X_{1} \beta_{01}\right)-\left[X_{1}^{\top} y-X_{1}^{\top} \mu\left(X_{1} \beta_{01}\right)\right]+n p_{\varpi}^{\prime}(\boldsymbol{\delta}) .
\end{aligned}
$$

Thus equation (1) is equivalent to $\varphi(\boldsymbol{\delta})=0$. Next, it can be shown that there exists a solution inside the hypercube $\mathcal{N}$ to satisfy $\varphi(\boldsymbol{\delta})=0$.

By second order Taylor expansion of $X_{1}^{\top} \mu\left(X_{1} \boldsymbol{\delta}\right)$ around $\beta_{01}$,

$$
\varphi(\boldsymbol{\delta})=\left[X_{1}^{\top} \Sigma\left(X_{1} \beta_{01}\right) X_{1}\right]\left(\boldsymbol{\delta}-\beta_{01}\right)-\left[\boldsymbol{\xi}_{1}-n p_{\varpi}^{\prime}(\boldsymbol{\delta})\right]+r,
$$

where $r=\left(r_{1}, \ldots, r_{s}\right)^{\top}$ are Lagrange remainders and for each $j=1, \ldots, s$,

$$
r_{j}=\frac{1}{2}\left(\delta-\beta_{01}\right)^{\top} \nabla^{2}\left[x_{j}^{\top} \mu\left(X_{1} \delta_{j}\right)\right]\left(\delta-\beta_{01}\right),
$$

where $\delta_{j}$ is some $s$-vector lying on the line segment joining $\delta$ and $\beta_{01}$.

By condition 4.2

$$
\begin{aligned}
\|r\|_{\infty} & \leq \max _{\delta \in \mathcal{N}} \max _{j=1}^{p} \lambda_{\max }\left[X_{1}^{\top} \operatorname{diag}\left\{\left|x_{j}\right| \circ\left|\mu^{\prime \prime}\left(X_{1} \delta\right)\right|\right\} X_{1}\right]\left\|\delta-\beta_{01}\right\|_{2}^{2} \\
& =O\left[s n^{1-2 \gamma_{0}} \log n\right]=O\left(n^{1-2 \gamma_{0}+\nu} \log n\right) .
\end{aligned}
$$

Let

$$
\bar{\varphi}(\boldsymbol{\delta})=\left[X_{1}^{\top} \Sigma\left(\theta_{0}\right) X_{1}\right]^{-1} \varphi(\boldsymbol{\delta})=\boldsymbol{\delta}-\beta_{10}+\mathbf{u},
$$

where $\mathbf{u}=-\left[X_{1}^{\top} \Sigma\left(\theta_{0}\right) X_{1}\right]^{-1}\left[\boldsymbol{\xi}_{1}-n p_{\varpi}^{\prime}(\delta)-r\right]$. For any $\delta \in \mathcal{N},\left|\delta_{j}\right| \geq\left|\beta_{0 j}\right|-d_{n}$, and thus

$$
\min _{j=1, \ldots, s}\left|\delta_{j}\right| \geq \min _{j=1, \ldots, s}\left|\beta_{0 j}\right|-d_{n}=d_{n} .
$$

By the monotonicity of $p_{\varpi}^{\prime}(\delta),\left\|p_{\varpi}^{\prime}(\delta)\right\|_{\infty} \leq p_{\varpi}^{\prime}\left(d_{n}\right)$, and thus with the definition of $\mathcal{E}_{1}$

$$
\left\|\boldsymbol{\xi}_{1}-n p_{\varpi}^{\prime}(\delta)\right\|_{\infty} \leq \sigma^{-1 / 2} n^{1 / 2} \sqrt{\log n}+n p_{\varpi}^{\prime}\left(d_{n}\right) .
$$

Therefore

$$
\begin{aligned}
\|\mathbf{u}\|_{\infty} & \leq\left\|\left[X_{1}^{\top} \Sigma\left(X \beta_{0}\right) X_{1}\right]^{-1}\right\|_{\infty}\left(\left\|\boldsymbol{\xi}_{1}-n p_{\varpi}^{\prime}(\delta)\right\|_{\infty}+\|r\|_{\infty}\right) \\
& =O\left[b_{s} n^{-1 / 2} \sqrt{\log n}+b_{s} p_{\varpi}^{\prime}\left(d_{n}\right)+b_{s} n^{\nu-2 \gamma_{0}} \log n\right] .
\end{aligned}
$$

By conditions 4.1 and 3.1, $\|\mathbf{u}\|_{\infty}=o\left(n^{-\gamma_{0}} \sqrt{\log n}\right)$.

For a constant $C>0$ and sufficiently large $n$, if $\delta_{j}-\beta_{j}=C n^{-\gamma_{0}} \sqrt{\log n}, \bar{\varphi}_{j}(\delta) \geq C n^{-\gamma_{0}} \sqrt{\log n}-\|\mathbf{u}\|_{\infty} \geq 0$. If $\delta_{j}-\beta_{j}=-C n^{-\gamma_{0}} \sqrt{\log n}, \bar{\varphi}_{j}(\delta) \leq-C n^{-\gamma_{0}} \sqrt{\log n}+\|\mathbf{u}\|_{\infty} \leq 0$. By the continuity of function $\bar{\varphi}(\boldsymbol{\delta})=$ $\left(\bar{\varphi}_{1}(\delta), \ldots, \bar{\varphi}_{s}(\delta)\right)$ and Miranda's existence theorem, there is a solution $\hat{\beta}_{1}$ for $\bar{\varphi}(\boldsymbol{\delta})=\mathbf{0}$ in $\mathcal{N}$, hence a solution for 
equation (1) in $\mathcal{N}$. Since the above conclusion holds for any $C>0$, we conclude that $\left\|\boldsymbol{\delta}-\beta_{01}\right\|_{\infty}=o\left(n^{-\gamma_{0}} \sqrt{\log n}\right)$.

\section{Step 2: Sparsity}

Let $\hat{\beta} \in \mathbf{R}^{p}$ with $\hat{\beta}_{1} \in \mathcal{N}$ as a solution for equation (1) and with $\hat{\beta}_{2}=0$. To prove the sparsity, we verify the equation (2) for $\hat{\beta}$.

$$
\left\|X_{2}^{\top}\left(y-\mu\left(X_{1} \hat{\beta}_{1}\right)\right)\right\|_{\infty}<n p_{\varpi}^{\prime}(0+)
$$

under the event $\mathcal{E}_{1} \cap \mathcal{E}_{2}$.

We first rewrite $X_{2}^{\top}\left(y-\mu\left(X_{1} \hat{\beta}_{1}\right)\right)$ in terms of $\boldsymbol{\xi}_{2}$ and another term:

$$
\begin{aligned}
X_{2}^{\top}\left(y-\mu\left(X_{1} \hat{\beta}_{1}\right)\right) & =X_{2}^{\top}\left(y-\mu\left(X_{1} \beta_{01}\right)\right)-X_{2}^{\top}\left(\mu\left(X_{1} \hat{\beta}_{1}\right)-\mu\left(X_{1} \beta_{01}\right)\right) \\
& =\boldsymbol{\xi}_{2}-X_{2}^{\top}\left(\mu\left(X_{1} \hat{\beta}_{1}\right)-\mu\left(X_{1} \beta_{01}\right)\right) .
\end{aligned}
$$

By condition 3.2, $\left\|\boldsymbol{\xi}_{2}\right\|_{\infty} \leq \sigma^{-1 / 2} n^{1 / 2+\alpha / 2} \sqrt{\log n}<(1-K) n p_{\varpi}^{\prime}(0+)$. Next we check the remainder term. A Taylor expansion of $X_{2}^{\top} \mu\left(X_{1} \hat{\beta}_{1}\right)$ around $\beta_{01}$ component-wise gives

$$
X_{2}^{\top} \mu\left(X_{1} \hat{\beta}_{1}\right)=X_{2}^{\top} \mu\left(X_{1} \beta_{01}\right)+X_{2}^{\top} \Sigma\left(\theta_{0}\right) X_{1}\left(\hat{\beta}_{1}-\beta_{01}\right)+w,
$$

where $w=\left(w_{s+1}, \ldots, w_{p}\right)$ and for each $j=(s+1), \ldots, p$,

$$
w_{j}=\frac{1}{2}\left(\hat{\beta}_{1}-\beta_{01}\right)^{\top} \nabla^{2}\left(x_{j}^{\top} \mu\left(X_{1} \delta_{j}\right)\right)\left(\hat{\beta}_{1}-\beta_{01}\right),
$$

where $\delta_{j}$ is some $s$-vector lying on the line segment joining $\hat{\beta}_{1}$ and $\beta_{01}$.

By condition 4.2

$$
\begin{aligned}
\|w\|_{\infty} & \leq \max _{\delta \in \mathcal{N}} \max _{j=1}^{p} \lambda_{\max }\left[X_{1}^{\top} \operatorname{diag}\left\{\left|x_{j}\right| \circ\left|\mu^{\prime \prime}\left(X_{1} \delta\right)\right|\right\} X_{1}\right]\left\|\hat{\beta}_{1}-\beta_{01}\right\|_{2}^{2} \\
& =O\left[s n^{1-2 \gamma_{0}} \log n\right]=O\left(n^{1-2 \gamma_{0}+\nu} \log n\right) .
\end{aligned}
$$

By the result in step 1,

$$
\hat{\beta}_{1}-\beta_{01}=\left(X_{1}^{\top} \Sigma\left(X \beta_{0}\right) X_{1}\right)^{-1}\left[X_{1}^{\top} \boldsymbol{\epsilon}-n p_{\varpi}^{\prime}\left(\hat{\beta}_{1}\right)-r\right] .
$$

By condition 3.2, $\left\|X_{2}^{\top} \Sigma\left(\theta_{0}\right) X_{1}\left[X_{1}^{\top} \Sigma\left(\theta_{0}\right) X_{1}\right]^{-1}\right\|_{\infty} \leq O\left(n^{\nu}\right)$,

$$
\begin{aligned}
& \left\|X_{2}^{\top}\left(\mu\left(X_{1} \hat{\beta}_{1}\right)-\mu\left(X_{1} \beta_{10}\right)\right)\right\|_{\infty} \\
= & \left\|X_{2}^{\top} \Sigma\left(X \beta_{0}\right) X_{1}\left(\hat{\beta}_{1}-\beta_{10}\right)+w\right\|_{\infty} \\
\leq & \left\|X_{2}^{\top} \Sigma\left(X \beta_{0}\right) X_{1}\left(X_{1}^{\top} \Sigma\left(X \beta_{0}\right) X_{1}\right)^{-1}\right\|_{\infty}\left[\left\|\boldsymbol{\xi}_{1}\right\|_{\infty}+n\left\|p_{\varpi}^{\prime}\left(\hat{\beta}_{1}\right)\right\|_{\infty}+\|r\|_{\infty}\right]+\|w\|_{\infty} \\
\leq & O\left[n^{\nu+1 / 2} \sqrt{\log (n)}+n^{1-2 \gamma_{0}+2 \nu} \log n+n^{1-2 \gamma_{0}+\nu} \log n\right] \\
& +\left\|X_{2}^{\top} \Sigma\left(X \beta_{0}\right) X_{1}\left(X_{1}^{\top} \Sigma\left(X \beta_{0}\right) X_{1}\right)^{-1}\right\|_{\infty}\left\|n p_{\varpi}^{\prime}\left(\hat{\beta}_{1}\right)\right\|_{\infty} .
\end{aligned}
$$


By condition 3.3, $\max \left(n^{1-2 \gamma_{0}+2 \nu} \log n, n^{\nu+1 / 2} \sqrt{\log n}\right)=o\left(n p_{\varpi}^{\prime}(0+)\right)$ and condition 3.2

$$
\begin{aligned}
& \left\|X_{2}^{\top} \Sigma\left(X \beta_{0}\right) X_{1}\left(X_{1}^{\top} \Sigma\left(X \beta_{0}\right) X_{1}\right)^{-1}\right\|_{\infty}\left\|n p_{\varpi}^{\prime}\left(\hat{\beta}_{1}\right)\right\|_{\infty} \\
\leq & \left\|X_{2}^{\top} \Sigma\left(X \beta_{0}\right) X_{1}\left(X_{1}^{\top} \Sigma\left(X \beta_{0}\right) X_{1}\right)^{-1}\right\|_{\infty} n p_{\varpi}^{\prime}\left(d_{n}\right) \\
\leq & K n p_{\varpi}^{\prime}(0+) .
\end{aligned}
$$

Therefore, we have

$$
\left\|X_{2}^{\top}(y-\mu(X \hat{\beta}))\right\|_{\infty}<(1-K)\left(n p_{\varpi}^{\prime}(0+)\right)+K n p_{\varpi}^{\prime}(0+)=n p_{\varpi}^{\prime}(0+) .
$$

Therefore for sufficient large $n$, equation (2) holds.

Finally, equation (3) would be satisfied by condition 4.4 for sufficiently large $n$.

\section{Lemma 1 (for proofs of the propositions 2 and 3)}

For condition 3.3, if $s=O\left(n^{\nu}\right) \ll \min \left(n^{\gamma_{0} / 2}(\log n)^{-1 / 4}, n^{-\gamma_{0}+1 / 2}\right)$, then

$$
\max \left(n^{-2 \gamma_{0}+2 \nu} \log n, n^{\nu-1 / 2} \sqrt{\log n}\right) \ll n^{-\gamma_{0}} \sqrt{\log n}=O\left(d_{n}\right) .
$$

Proof:

$$
\begin{aligned}
& \text { If } 1 / 3<\gamma_{0}<1 / 2 \text {, then } \min \left(n^{\gamma_{0} / 2}(\log n)^{-1 / 4}, n^{-\gamma_{0}+1 / 2}\right)=n^{-\gamma_{0}+1 / 2} \\
& \max \left(n^{-2 \gamma_{0}+2 \nu} \log n, n^{\nu-1 / 2} \sqrt{\log n}\right)=\max \left(s^{2} n^{-2 \gamma_{0}} \log n, s n^{-1 / 2} \sqrt{\log n}\right) \\
& \ll \max \left(n^{1-4 \gamma_{0}} \log n, n^{-\gamma_{0}} \sqrt{\log n}\right) \\
& =n^{-\gamma_{0}} \sqrt{\log n}=O\left(d_{n}\right) \text {. }
\end{aligned}
$$

If $0 \leq \gamma_{0} \leq 1 / 3$, then $\min \left(n^{\gamma_{0} / 2}(\log n)^{-1 / 4}, n^{-\gamma_{0}+1 / 2}\right)=n^{\gamma_{0} / 2}(\log n)^{-1 / 4}$,

$$
\begin{aligned}
\max \left(n^{-2 \gamma_{0}+2 \nu} \log n, n^{\nu-1 / 2} \sqrt{\log n}\right) & =\max \left(s^{2} n^{-2 \gamma_{0}} \log n, s n^{-1 / 2} \sqrt{\log n}\right) \\
& \ll \max \left(n^{-\gamma_{0}} \sqrt{\log n}, n^{\gamma_{0} / 2-1 / 2}(\log n)^{1 / 4}\right) \\
& =n^{-\gamma_{0}} \sqrt{\log n}=O\left(d_{n}\right) .
\end{aligned}
$$

Therefore, $\max \left(n^{-2 \gamma_{0}+2 \nu} \log n, n^{\nu-1 / 2} \sqrt{\log n}\right) \ll n^{-\gamma_{0}} \sqrt{\log n}=O\left(d_{n}\right)$.

\section{Lemma 2 (for proofs of propositions 2 and 3 )}

For condition 3.3, if $s \ll \min \left(n^{\gamma_{0} / 2-\gamma_{s} / 2}(\log n)^{-1 / 4}, n^{-\gamma_{0}-\gamma_{s}+1 / 2}\right)$, then

$$
\max \left(n^{-2 \gamma_{0}+2 \nu} \log n, n^{\nu-1 / 2} \sqrt{\log n}\right) \ll n^{-\gamma_{0}-\gamma_{s}} \sqrt{\log n}=O\left(b_{s}^{-1} d_{n}\right) .
$$

Proof:

$$
\begin{aligned}
& \text { If } \gamma_{0}+\gamma_{s} / 3>1 / 3 \text {, then } \min \left(n^{\gamma_{0} / 2-\gamma_{s} / 2}(\log n)^{-1 / 4}, n^{-\gamma_{0}-\gamma_{s}+1 / 2}\right)=n^{-\gamma_{0}-\gamma_{s}+1 / 2} \text {, and } \\
& \max \left(n^{-2 \gamma_{0}+2 \nu} \log n, n^{\nu-1 / 2} \sqrt{\log n}\right)=\max \left(s^{2} n^{-2 \gamma_{0}} \log n, s n^{-1 / 2} \sqrt{\log n}\right) \\
& \ll \max \left(n^{1-4 \gamma_{0}-2 \gamma_{s}} \log n, n^{-\gamma_{0}-\gamma_{s}} \sqrt{\log n}\right) \\
& =n^{-\gamma_{0}-\gamma_{s}} \sqrt{\log n}=O\left(b_{s}^{-1} d_{n}\right) \text {. }
\end{aligned}
$$


If $0 \leq \gamma_{0}+\gamma_{s} / 3 \leq 1 / 3$, then $\min \left(n^{\frac{\gamma_{0}}{2}-\frac{\gamma_{s}}{2}}(\log n)^{-1 / 4}, n^{-\gamma_{0}-\gamma_{s}+1 / 2}\right)=n^{\gamma_{0} / 2-\gamma_{s} / 2}(\log n)^{-1 / 4}$, and

$$
\begin{aligned}
\max \left(n^{-2 \gamma_{0}+2 \nu} \log n, n^{\nu-1 / 2} \sqrt{\log n}\right) & =\max \left(s^{2} n^{-2 \gamma_{0}} \log n, s n^{-1 / 2} \sqrt{\log n}\right) \\
& \ll \max \left(n^{-\gamma_{0}-\gamma_{s}} \sqrt{\log n}, n^{\gamma_{0} / 2-\gamma_{s} / 2-1 / 2}(\log n)^{1 / 4}\right) . \\
& =n^{-\gamma_{0}-\gamma_{s}} \sqrt{\log n}=O\left(b_{s}^{-1} d_{n}\right) .
\end{aligned}
$$

Thus $\max \left(n^{-2 \gamma_{0}+2 \nu} \log n, n^{\nu-1 / 2} \sqrt{\log n}\right) \ll n^{-\gamma_{0}-\gamma_{s}} \sqrt{\log n}=O\left(b_{s}^{-1} d_{n}\right)$

\section{Proof of Proposition 1}

For SCAD:

- Given $d_{n} \gg \eta_{p}$ and $s \ll \eta_{d}$, we will show that if $\lambda_{n}=O\left(d_{n}\right)$ and $d_{n} \geq a \lambda_{n}$ (more precisely, $d_{n} \geq a \lambda_{n}$ for $\operatorname{SCAD}_{\lambda_{n}}$ or $d_{n} \geq a_{n} \lambda_{n}$ for $\left.\operatorname{SCAD}_{\lambda_{n}, a_{n}}\right)$, conditions 3.1-3.3 and 4.4 are satisfied.

- Since $d_{n} \geq a \lambda_{n}, p_{\operatorname{SCAD}_{\lambda_{n}}}^{\prime}\left(d_{n}\right)=p_{\operatorname{SCAD}_{\lambda_{n}, a_{n}}}^{\prime}\left(d_{n}\right)=0$. Therefore condition 3.1 is satisfied.

- Because $p_{\operatorname{SCAD}_{\lambda_{n}}}^{\prime}(0+)=p_{\operatorname{SCAD}_{\lambda_{n}, a_{n}}}^{\prime}(0+)=\lambda_{n}$, condition 3.3 becomes

$$
\lambda_{n}>\frac{\sigma^{-1 / 2}}{(1-K)} n^{-1 / 2+\alpha / 2} \sqrt{\log n} \text { and } \lambda_{n} \gg \max \left(n^{-2 \gamma_{0}+2 \nu} \log n, n^{\nu-1 / 2} \sqrt{\log n}\right) .
$$

First, $\lambda_{n}>\frac{\sigma^{-1 / 2}}{(1-K)} n^{-1 / 2+\alpha / 2} \sqrt{\log n}$ by our choice of $\lambda_{n}=O\left(d_{n}\right)$, and the assumption $d_{n} \gg n^{-1 / 2+\alpha / 2} \sqrt{\log n}$. Next, $\lambda_{n} \gg \max \left(n^{-2 \gamma_{0}+2 \nu} \log n, n^{\nu-1 / 2} \sqrt{\log n}\right)$ is satisfied by Lemma 1 , and the choice of $\lambda_{n}=O\left(d_{n}\right)$. Therefore condition 3.3 is satisfied.

- For either $\operatorname{SCAD}_{\lambda_{n}}$ or $\operatorname{SCAD}_{\lambda_{n}, a_{n}}$, we have $p_{\varpi}^{\prime}(0+) / p_{\varpi}^{\prime}\left(d_{n}\right)=\infty$ because $p_{\varpi}^{\prime}(0+)>0$ and $p_{\varpi}^{\prime}\left(d_{n}\right)=0$. Therefore condition 3.2 is satisfied.

- For any $\delta=\left(\delta_{1}, \ldots, \delta_{s}\right)^{\top} \in \mathcal{N}_{0} \equiv\left\{\boldsymbol{\delta} \in \mathbf{R}^{s}:\left\|\boldsymbol{\delta}-\beta_{01}\right\|_{\infty} \leq d_{n}\right\}$, we have $\left|\delta_{j}\right| \geq d_{n} \geq a \lambda_{n}$, and thus $\kappa\left(p_{\varpi}, \delta_{j}\right)=0$ for either $\operatorname{SCAD}_{\lambda_{n}}$ or $\mathrm{SCAD}_{\lambda_{n}, a_{n}}$. Therefore condition 4.4 is satisfied.

- Given $d_{n} \ll O\left(n^{-1 / 2+\alpha / 2} \sqrt{\log n}\right)$,

- Condition 3.3 requires $\lambda_{n}>\frac{\sigma^{-1 / 2}}{(1-K)} n^{-1 / 2+\alpha / 2} \sqrt{\log n}$. Given $d_{n} \ll n^{-1 / 2+\alpha / 2} \sqrt{\log n}$, we have $d_{n} \ll$ $\lambda_{n}$. Therefore, $p_{\mathrm{SCAD}_{\lambda_{n}}}^{\prime}\left(d_{n}\right)=p_{\mathrm{SCAD}_{\lambda_{n}, a_{n}}}^{\prime}\left(d_{n}\right)=\lambda_{n}$.

- Condition 3.1 requires $p_{\varpi}^{\prime}\left(d_{n}\right)=\lambda_{n} \ll d_{n}$ since $b_{s}^{-1} d_{n} \ll d_{n}$.

Clearly, no such $\lambda_{n}$ exists to satisfy $d_{n} \gg \lambda_{n}$ and $d_{n} \ll \lambda_{n}$ or $d_{n}<\lambda_{n}$ simultaneously.

For $\mathrm{MCP}_{\lambda_{n}}$ :

- Given $d_{n} \gg \frac{\sigma^{-1 / 2}}{(1-K)} n^{-1 / 2+\alpha / 2} \sqrt{\log n}$ and $s \ll \min \left(n^{\gamma_{0} / 2}(\log n)^{-1 / 4}, n^{-\gamma_{0}+1 / 2}\right)$, we will show that if $\lambda_{n}=$ $O\left(d_{n}\right)$ and $d_{n} \geq a \lambda_{n}$, conditions 3.1-3.3 and 4.4 are satisfied.

- Since $d_{n} \geq a \lambda_{n}, p_{\mathrm{MCP}_{\lambda_{n}}}^{\prime}\left(d_{n}\right)=0$. Therefore condition 3.1 is satisfied. 
- Because $p_{\mathrm{MCP}_{\lambda_{n}}}^{\prime}(0+)=\lambda_{n}$, condition 3.3 becomes

$$
\lambda_{n}>\frac{\sigma^{-1 / 2}}{(1-K)} n^{-1 / 2+\alpha / 2} \sqrt{\log n} \text { and } \lambda_{n} \gg \max \left(n^{-2 \gamma_{0}+2 \nu} \log n, n^{\nu-1 / 2} \sqrt{\log n}\right) .
$$

First, $\lambda_{n}>\frac{\sigma^{-1 / 2}}{(1-K)} n^{-1 / 2+\alpha / 2} \sqrt{\log n}$ by our choice of $\lambda_{n}$. Next, by Lemma 1 ,

$\lambda_{n} \gg \max \left(n^{-2 \gamma_{0}+2 \nu} \log n, n^{\nu-1 / 2} \sqrt{\log n}\right)$ because $\lambda_{n}=O\left(d_{n}\right)$. Therefore condition 3.3 is satisfied.

- For $\mathrm{MCP}_{\lambda_{n}}, p_{\mathrm{MCP}}^{\prime}(0+) / p_{\mathrm{MCP}}^{\prime}\left(d_{n}\right)=\infty$ because $p_{\mathrm{MCP}}^{\prime}(0+)>0$ and $p_{\mathrm{MCP}}^{\prime}\left(d_{n}\right)=0$. Therefore condition 3.2 is satisfied.

- Because $d_{n} \geq a \lambda_{n}, \kappa\left(p_{\varpi}, \delta\right)=0$ for any $\delta \in \mathcal{N}_{0} \equiv\left\{\boldsymbol{\delta} \in \mathbf{R}^{s}:\left\|\boldsymbol{\delta}-\beta_{01}\right\|_{\infty} \leq d_{n}\right\}$. Thus condition 4.4 is satisfied.

- Given $d_{n} \ll O\left(n^{-1 / 2+\alpha / 2} \sqrt{\log n}\right)$,

- Condition 3.3 requires $\lambda_{n}>\frac{\sigma^{-1 / 2}}{(1-K)} n^{-\frac{1}{2}+\frac{\alpha}{2}} \sqrt{\log n}$. Given $d_{n} \ll n^{-\frac{1}{2}+\frac{\alpha}{2}} \sqrt{\log n}$, it leads $d_{n} \ll \lambda_{n}$ or $d_{n}<\lambda_{n}$. Therefore, $p_{\mathrm{MCP}_{\lambda_{n}}}^{\prime}\left(d_{n}\right)=\lambda_{n}$.

- Condition 3.1 requires $p_{\varpi}^{\prime}\left(d_{n}\right)=\lambda_{n} \ll d_{n}$ since $b_{s}^{-1} d_{n} \ll d_{n}$.

Clearly, no such $\lambda_{n}$ exists to satisfy both conditions simultaneously.

\section{Proof of Proposition 2}

For $\mathrm{MCP}_{\lambda_{n}, a_{n}}$, we will show that if $\left(\lambda_{n}, a_{n}\right)$ satisfy $\lambda_{n}>\eta_{p}, \lambda_{n} \gg \max \left(n^{-2 \gamma_{0}+2 \nu} \log n, n^{\nu-1 / 2} \sqrt{\log n}\right)$, and $a_{n} \lambda_{n}<d_{n}$, conditions 3.1-3.3 and 4.4 are satisfied.

- Since $d_{n} \geq a_{n} \lambda_{n}, p_{\mathrm{MCP}_{\lambda_{n}}, a_{n}}^{\prime}\left(d_{n}\right)=0$. Therefore, condition 3.1 is satisfied.

- Because $p_{\mathrm{MCP}_{\lambda_{n}, a_{n}}}^{\prime}(0+)=\lambda_{n}$, condition 3.3 becomes

$$
\lambda_{n}>\frac{\sigma^{-1 / 2}}{(1-K)} n^{-1 / 2+\alpha / 2} \sqrt{\log n} \text { and } \lambda_{n} \gg \max \left(n^{-2 \gamma_{0}+2 \nu} \log n, n^{\nu-1 / 2} \sqrt{\log n}\right) .
$$

By our choice of $\lambda_{n}$, condition 3.3 is satisfied.

- For $\mathrm{MCP}_{\lambda_{n}, a_{n}}, p_{\mathrm{MCP}}^{\prime}(0+) / p_{\mathrm{MCP}}^{\prime}\left(d_{n}\right)=\infty$ because $p_{\mathrm{MCP}}^{\prime}(0+)>0$ and $p_{\mathrm{MCP}}^{\prime}\left(d_{n}\right)=0$. Therefore condition 3.2 is satisfied.

- Because $d_{n} \geq a \lambda_{n}, \kappa\left(p_{\varpi}, \delta\right)=0$ for any $\delta \in \mathcal{N}_{0} \equiv\left\{\boldsymbol{\delta} \in \mathbf{R}^{s}:\left\|\boldsymbol{\delta}-\beta_{01}\right\|_{\infty} \leq d_{n}\right\}$. Thus condition 4.4 is satisfied.

\section{Proof of Proposition 3}

\section{For $\operatorname{SICA}_{\lambda_{n}}$ :}

- $p_{\mathrm{SICA}_{\lambda_{n}}}^{\prime}(0+)=\lambda_{n}(1+1 / \tau)=O\left(\lambda_{n}\right)$ and $p_{\mathrm{SICA}_{\lambda_{n}}}^{\prime}\left(d_{n}\right)=\frac{\lambda_{n} \tau(\tau+1)}{\left(d_{n}+\tau\right)^{2}}=O\left(\lambda_{n}\right)$.

Because $s \ll \min \left(n^{\gamma_{0} / 2-\gamma_{s} / 2}(\log n)^{-1 / 4}, n^{-\gamma_{0}-\gamma_{s}+1 / 2}\right)$ and $\alpha<1-2 \gamma_{0}-2 \gamma_{s}$, we have

$$
\max \left(n^{-1 / 2+\alpha / 2} \sqrt{\log n}, n^{-2 \gamma_{0}+2 \nu} \log n, n^{\nu-1 / 2} \sqrt{\log n}\right) \ll n^{-\gamma_{s}-\gamma_{0}} \sqrt{\log n}
$$

by Lemma 2. In addition, given $\left\|X_{2}^{\top} \Sigma\left(\theta_{0}\right) X_{1}\left(X_{1}^{\top} \Sigma\left(\theta_{0}\right) X_{1}\right)^{-1}\right\|_{\infty} \leq K\left(d_{n} / \tau+1\right)^{2}$, we will show that if

$$
\max \left(n^{-1 / 2+\alpha / 2} \sqrt{\log n}, n^{-2 \gamma_{0}+2 \nu}(\log n)^{2}, n^{\nu-1 / 2} \sqrt{\log n}\right) \ll \lambda_{n} \ll n^{-\gamma_{s}-\gamma_{0}} \sqrt{\log n},
$$

conditions 3.1-3.3 and 4.4 are satisfied. 
- Since $p_{\mathrm{SICA}_{\lambda_{n}}}^{\prime}\left(d_{n}\right)=O\left(\lambda_{n}\right) \ll n^{-\gamma_{s}-\gamma_{0}} \sqrt{\log n}$, condition 3.1 is satisfied.

- Because $p_{\mathrm{SICA}_{\lambda_{n}}}^{\prime}(0+)=O\left(\lambda_{n}\right)$ by the choice of $\lambda_{n}$, condition 3.3 is satisfied by

$$
\max \left(n^{-1 / 2+\alpha / 2} \sqrt{\log n}, n^{-2 \gamma_{0}+2 \nu}(\log n)^{2}, n^{\nu-1 / 2} \sqrt{\log n}\right) \ll \lambda_{n}
$$

- Since $p_{\mathrm{SICA}_{\lambda_{n}}}^{\prime}(0+) / p_{\mathrm{SICA}_{\lambda_{n}}}^{\prime}\left(d_{n}\right)=\left(d_{n} / \tau+1\right)^{2}$, condition 3.2 is satisfied by

$$
\left\|X_{2}^{\top} \Sigma\left(\theta_{0}\right) X_{1}\left(X_{1}^{\top} \Sigma\left(\theta_{0}\right) X_{1}\right)^{-1}\right\|_{\infty} \leq K\left(\frac{d_{n}}{\tau}+1\right)^{2} .
$$

- Because $p_{\mathrm{SICA}_{\lambda_{n}}}^{\prime \prime}\left(d_{n}\right)=O\left(\lambda_{n}\right)=o(1)$, condition 4.4 is satisfied.

- Given $d_{n} \ll O\left(n^{-1 / 2+\alpha / 2} \sqrt{\log n}\right)$,

- Condition 3.3 requires $p_{\mathrm{SICA}_{\lambda_{n}}}^{\prime}(0+)=O\left(\lambda_{n}\right)>\frac{\sigma^{-1 / 2}}{(1-K)} n^{-\frac{1}{2}+\frac{\alpha}{2}} \sqrt{\log n}$. Given $d_{n} \ll n^{-\frac{1}{2}+\frac{\alpha}{2}} \sqrt{\log n}$, it leads $d_{n} \ll \lambda_{n}$. Therefore, $p_{\mathrm{SICA} \lambda_{n}}^{\prime}\left(d_{n}\right)=O\left(\lambda_{n}\right)$.

- Condition 3.1 requires $p_{\varpi}^{\prime}\left(d_{n}\right)=\lambda_{n} \ll d_{n}$ since $b_{s}^{-1} d_{n} \ll d_{n}$.

Clearly, no such $\lambda_{n}$ exists to satisfy both conditions simultaneously.

For $\log _{\lambda_{n}}$ :

- $p_{\mathrm{Log} \lambda_{n}}^{\prime}(0+)=\lambda_{n} / \tau=O\left(\lambda_{n}\right)$ and $p_{\log _{\lambda_{n}}}^{\prime}\left(d_{n}\right)=\lambda_{n} /\left(d_{n}+\tau\right)=O\left(\lambda_{n}\right)$.

Given $\alpha<1-2 \gamma_{0}-2 \gamma_{s}$ and $s \ll \min \left(n^{\gamma_{0} / 2-\gamma_{s} / 2}(\log n)^{-1 / 4}, n^{-\gamma_{0}-\gamma_{s}+1 / 2}\right)$, by Lemma 2 , we have

$$
\max \left(n^{-1 / 2+\alpha / 2} \sqrt{\log n}, n^{-2 \gamma_{0}+2 \nu} \log n, n^{\nu-1 / 2} \sqrt{\log n}\right) \ll n^{-\gamma_{s}-\gamma_{0}} \sqrt{\log n} .
$$

Given the additional condition $\left\|X_{2}^{\top} \Sigma\left(\theta_{0}\right) X_{1}\left(X_{1}^{\top} \Sigma\left(\theta_{0}\right) X_{1}\right)^{-1}\right\|_{\infty} \leq K\left(d_{n} / \tau+1\right)$, we will show that if

$$
\max \left(n^{-1 / 2+\alpha / 2} \sqrt{\log n}, n^{-2 \gamma_{0}+2 \nu} \log n, n^{\nu-1 / 2} \sqrt{\log n}\right) \ll \lambda_{n} \ll n^{-\gamma_{s}-\gamma_{0}} \sqrt{\log n},
$$

conditions 3.1-3.3 and 4.4 are satisfied.

- Since $p_{\log _{\lambda_{n}}}^{\prime}\left(d_{n}\right)=O\left(\lambda_{n}\right) \ll n^{-\gamma_{s}-\gamma_{0}} \sqrt{\log n}$ by the choice of $\lambda_{n}$, condition 3.1 is satisfied.

- Because $p_{\mathrm{Log}_{\lambda_{n}}}^{\prime}(0+)=O\left(\lambda_{n}\right)$, by the choice of $\lambda_{n}$, condition 3.3 is satisfied by

$$
\max \left(n^{-1 / 2+\alpha / 2} \sqrt{\log n}, n^{-2 \gamma_{0}+2 \nu} \log n, n^{\nu-1 / 2} \sqrt{\log n}\right) \ll \lambda_{n} .
$$

- Since $p_{\log _{\lambda_{n}}}^{\prime}(0+) / p_{\log _{\lambda_{n}}}^{\prime}\left(d_{n}\right)=d_{n} / \tau+1$, condition 3.2 is satisfied given

$$
\left\|X_{2}^{\top} \Sigma\left(\theta_{0}\right) X_{1}\left(X_{1}^{\top} \Sigma\left(\theta_{0}\right) X_{1}\right)^{-1}\right\|_{\infty} \leq K\left(\frac{d_{n}}{\tau}+1\right) .
$$

- Because $p_{\log _{\lambda_{n}}}^{\prime \prime}\left(d_{n}\right)=O\left(\lambda_{n}\right)=o(1)$, condition 4.4 is satisfied.

- Given $d_{n} \ll O\left(n^{-1 / 2+\alpha / 2} \sqrt{\log n}\right)$,

- Condition 3.3 requires $p_{\text {Log }_{\lambda_{n}}}^{\prime}(0+)=O\left(\lambda_{n}\right)>\frac{\sigma^{-1 / 2}}{(1-K)} n^{-\frac{1}{2}+\frac{\alpha}{2}} \sqrt{\log n}$. Given $d_{n} \ll n^{-\frac{1}{2}+\frac{\alpha}{2}} \sqrt{\log n}$, it leads $d_{n} \ll \lambda_{n}$. Therefore, $p_{\log _{\lambda_{n}}^{\prime}}^{\prime}\left(d_{n}\right)=O\left(\lambda_{n}\right)$.

- Condition 3.1 requires $p_{\varpi}^{\prime}\left(d_{n}\right)=\lambda_{n} \ll d_{n}$ since $b_{s}^{-1} d_{n} \ll d_{n}$.

Clearly, no such $\lambda_{n}$ exists to satisfy both conditions simultaneously. 


\section{Proof of Proposition 4}

For $\operatorname{SICA}_{\lambda_{n}, a_{n}}$ :

- Let $\lambda_{n}=O\left(n^{\gamma_{\lambda}}\right)$ and $\tau_{n}=O\left(n^{\gamma_{\tau}}\right)$. Given $0<\alpha<1$ and $\nu \leq \gamma_{0}$, we will show that if $\gamma_{\tau}<-2 \gamma_{0}-\gamma_{s}<$ $\gamma_{\lambda}<-\gamma_{0}$, conditions 3.1-3.3 and 4.4 are satisfied.

- Given $\gamma_{\tau}<-\gamma_{0}, \exists$ constant $C$ such that $d_{n}+\tau_{n} \geq C^{-1} n^{-\gamma_{0}}$. Therefore

$$
\begin{aligned}
p_{\mathrm{SICA}_{\lambda_{n}, a_{n}}}^{\prime}\left(d_{n}\right) & =\frac{\lambda_{n} \tau_{n}\left(\tau_{n}+1\right)}{\left(d_{n}+\tau_{n}\right)^{2}} \leq C^{2} n^{2 \gamma_{0}} \lambda_{n} \tau_{n}\left(\tau_{n}+1\right)=O\left(n^{2 \gamma_{0}+\gamma_{\lambda}+\gamma_{\tau}}\right) \\
\kappa\left(p_{\varpi}, \delta\right) & =\left|p_{\mathrm{SICA}_{\lambda_{n}, \tau_{n}}}^{\prime \prime}\left(d_{n}\right)\right|=\frac{2 \lambda_{n} \tau_{n}\left(\tau_{n}+1\right)}{\left(d_{n}+\tau_{n}\right)^{3}} \leq 2 C^{3} n^{3 \gamma_{0}} \lambda_{n} \tau_{n}\left(\tau_{n}+1\right) \\
& =O\left(n^{3 \gamma_{0}+\gamma_{\lambda}+\gamma_{\tau}}\right) .
\end{aligned}
$$

- $p_{\mathrm{SICA}_{\lambda_{n}, a_{n}}}^{\prime}\left(d_{n}\right)=O\left(n^{2 \gamma_{0}+\gamma_{\lambda}+\gamma_{\tau}}\right) \ll b_{s}^{-1} d_{n}=O\left(n^{-\gamma_{s}-\gamma_{0}} \sqrt{\log n}\right)$ by the choice of $\lambda_{n}, \tau_{n}$ with $\gamma_{\lambda}+$ $\gamma_{\tau}<-3 \gamma_{0}-\gamma_{s}$. Therefore, condition 3.1 is satisfied.

- Since $p_{\mathrm{SICA}_{\lambda_{n}, \tau_{n}}}^{\prime}(0+)=O\left(n^{\gamma_{\lambda}-\gamma_{\tau}}\right)$, condition 3.3 becomes

$$
n^{\gamma_{\lambda}-\gamma_{\tau}} \gg \max \left(n^{-2 \gamma_{0}+2 \nu} \log n, n^{\nu-1 / 2} \sqrt{\log n}\right)
$$

and

$$
n^{\gamma_{\lambda}-\gamma_{\tau}}>\frac{\sigma^{-1 / 2}}{(1-K)} n^{-1 / 2+\alpha / 2} \sqrt{\log n}
$$

Given $0 \leq \alpha<1$ and $\nu<\gamma_{0}<1 / 2$ (conditions 2.1 and 2.2),

$$
\max \left(\frac{\sigma^{-1 / 2}}{(1-K)} n^{-1 / 2+\alpha / 2} \sqrt{\log n}, n^{-2 \gamma_{0}+2 \nu} \log n, n^{\nu-1 / 2} \sqrt{\log n}\right) \ll \log n
$$

and $n^{\gamma_{\lambda}-\gamma_{\tau}} \gg \log n$ by $\gamma_{\lambda}-\gamma_{\tau}>0$. Thus condition 3.3 is satisfied.

- By conditions 2.1 and 2.2, $0 \leq \nu<\gamma_{0}<1 / 2$, thus $\gamma_{\tau}<-2 \gamma_{0}<-\gamma_{0}-\nu / 2$. Thus $\frac{p_{\mathrm{SICA}_{\lambda_{n}}, \tau_{n}}^{\prime}(0+)}{p_{\mathrm{SICA}_{\lambda_{n}}, \tau_{n}}\left(d_{n}\right)}=$ $\left(d_{n} / \tau_{n}+1\right)^{2}=O\left(n^{-2 \gamma_{0}-2 \gamma_{\tau}} \log n\right) \gg O\left(n^{v}\right)$. Therefore condition 3.2 is satisfied

- Condition 4.4 is fulfilled by $\kappa\left(p_{\varpi}, \delta\right)=\left|p_{\mathrm{SICA}_{\lambda_{n}, \tau_{n}}^{\prime \prime}}\left(d_{n}\right)\right|=O\left(n^{3 \gamma_{0}+\gamma_{\lambda}+\gamma_{\tau}}\right)=o(1)$ because $\gamma_{\tau}+\gamma_{\lambda}<$ $-3 \gamma_{0}$.

For $\log _{\lambda_{n}, a_{n}}$ :

- Given $0<\alpha<1$ and $\nu \leq \gamma_{0}$, we will show that if $\gamma_{\tau}<\gamma_{\lambda}<-2 \gamma_{0}-\gamma_{s}$, conditions 3.1-3.3 and 4.4 are satisfied. Given $\gamma_{\tau}<-\gamma_{0}, \exists$ constant $C$ such that $d_{n}+\tau_{n} \geq C^{-1} n^{-\gamma_{0}}$. Therefore

$$
\begin{aligned}
p_{\mathrm{Log}_{\lambda_{n}, \tau_{n}}}^{\prime}\left(d_{n}\right) & =\lambda_{n} /\left(d_{n}+\tau_{n}\right) \leq C n^{\gamma_{0}} \lambda_{n}=O\left(n^{\gamma_{0}+\gamma_{\lambda}}\right) \\
\kappa\left(p_{\varpi}, \delta\right) & =\left|p_{\log _{\lambda_{n}, \tau_{n}}}^{\prime \prime}\left(d_{n}\right)\right|=\lambda_{n} /\left(d_{n}+\tau_{n}\right)^{2} \leq C^{2} n^{2 \gamma_{0}} \lambda_{n}=O\left(n^{2 \gamma_{0}+\gamma_{\lambda}}\right) .
\end{aligned}
$$

- $p_{\mathrm{Log}_{\lambda_{n}, \tau_{n}}}^{\prime}\left(d_{n}\right)=O\left(n^{\gamma_{0}+\gamma_{\lambda}}\right) \ll b_{s}^{-1} d_{n}=O\left(n^{-\gamma_{s}-\gamma_{0}} \sqrt{\log n}\right)$ by the choice of $\lambda_{n}$ with $\gamma_{\lambda}<-2 \gamma_{0}-\gamma_{s}$. Therefore, condition 3.1 is satisfied.

- Since $p_{\log _{\lambda_{n}}}^{\prime}(0+)=\lambda_{n} / \tau_{n}=O\left(n^{\gamma_{\lambda}-\gamma_{\tau}}\right)$. Condition 3.3 becomes

$$
n^{\gamma_{\lambda}-\gamma_{\tau}} \gg \max \left(n^{-2 \gamma_{0}+2 \nu} \log n, n^{\nu-1 / 2} \sqrt{\log n}\right)
$$


and

$$
n^{\gamma_{\lambda}-\gamma_{\tau}}>\frac{\sigma^{-1 / 2}}{(1-K)} n^{-1 / 2+\alpha / 2} \sqrt{\log n} .
$$

Given $0 \leq \alpha<1$ and $\nu<\gamma_{0}<1 / 2$ (conditions 2.1 and 2.2),

$$
\max \left(\frac{\sigma^{-1 / 2}}{(1-K)} n^{-1 / 2+\alpha / 2} \sqrt{\log n}, n^{-2 \gamma_{0}+2 \nu} \log n, n^{\nu-1 / 2} \sqrt{\log n}\right) \ll \log n .
$$

Because $\gamma_{\lambda}-\gamma_{\tau}>0, n^{\gamma_{\lambda}-\gamma_{\tau}} \gg \log n$. Thus condition 3.3 is satisfied.

- By conditions 2.1 and 2.2, $0 \leq \nu<\gamma_{0}<1 / 2$, thus $\gamma_{\tau}<-2 \gamma_{0}<-\gamma_{0}-\nu$. Thus $\frac{p_{\log _{\lambda_{n}, \tau_{n}}^{\prime}}(0+)}{p_{\log _{\lambda_{n}, \tau_{n}}}^{\prime}\left(d_{n}\right)}=$ $d_{n} / \tau_{n}+1=O\left(n^{-\gamma_{0}-\gamma_{\tau}} \sqrt{\log n}\right) \gg O\left(n^{v}\right)$. Therefore condition 3.2 is satisfied

- Condition 4.4 is fulfilled by $\kappa\left(p_{\varpi}, \delta\right)=\left|p_{\log _{\lambda_{n}, \tau_{n}}}^{\prime \prime}\left(d_{n}\right)\right|=O\left(n^{2 \gamma_{0}+\gamma_{\lambda}}\right)=o(1)$ because $\gamma_{\lambda}<-2 \gamma_{0}$.

\section{Proof of Corollary 1}

Given $d_{n} \ll \eta_{p}$ :

- For $\mathrm{MCP}_{\lambda_{n}, a_{n}}$ :

- Condition 3.3 requires $\lambda_{n}>\eta_{p}$.

- If $a_{n}$ is tuned such that $d_{n}<a_{n} \lambda_{n}$, then $p_{\varpi}^{\prime}\left(d_{n}\right)=\lambda_{n}+d_{n} / a_{n}$. Ccondition 3.3 requires $\lambda_{n}>\eta_{p}$ so that condition 3.1 cannot be satisfied due to $p_{\varpi}^{\prime}\left(d_{n}\right)>\eta_{p} \gg d_{n}$.

- If $a_{n}$ is tuned such that $d_{n} \geq a_{n} \lambda_{n}$, then $p_{\varpi}^{\prime}\left(d_{n}\right)=0$. Therefore, condition 3.1 can be satisfied. This restricts the valid range of $a_{n}: a_{n}<d_{n} / \lambda_{n}<d_{n} / \eta_{p}=o(1)$.

- For $\operatorname{SICA}_{\lambda_{n}, \tau_{n}}$ :

- Condition 3.3 requires $p_{\varpi}^{\prime}(0+)>\eta_{p}$.

- Note that $p_{\varpi}^{\prime}\left(d_{n}\right)$ of SICA can be expressed as $p_{\varpi}^{\prime}\left(d_{n}\right)=p_{\varpi}^{\prime}(0+) /\left(d_{n} / \tau_{n}+1\right)^{2}$. Condition 3.1 requires $p_{\varpi}^{\prime}\left(d_{n}\right)=p_{\varpi}^{\prime}(0+) /\left(d_{n} / \tau_{n}+1\right)^{2} \ll d_{n}$. Combining condition with 3.3 , it leads that $\eta_{p} /\left(d_{n} / \tau_{n}+1\right)^{2} \ll$ $p_{\varpi}^{\prime}(0+) /\left(d_{n} / \tau_{n}+1\right)^{2} \ll d_{n}$. Note that condition 3.2 requires $d_{n} / \tau_{n} \rightarrow \infty$ as shown in proof of proposition 4 so that $O\left(d_{n} / \tau_{n}+1\right)=O\left(d_{n} / \tau_{n}\right)$. Therefore, the valid range of $\tau_{n}$ is restricted as $\tau_{n}<d_{n}^{3 / 2} / \eta_{p}^{1 / 2}=o(1)$.

- For $\log _{\lambda_{n}, \tau_{n}}$ :

- Condition 3.3 requires $p_{\varpi}^{\prime}(0+)>\eta_{p}$.

- Note that $p_{\varpi}^{\prime}\left(d_{n}\right)$ of Log can be expressed as $p_{\varpi}^{\prime}\left(d_{n}\right)=p_{\varpi}^{\prime}(0+) /\left(d_{n} / \tau_{n}+1\right)$. Condition 3.1 requires $p_{\varpi}^{\prime}\left(d_{n}\right)=p_{\varpi}^{\prime}(0+) /\left(d_{n} / \tau_{n}+1\right) \ll d_{n}$. Combined with condition 3.3, it leads that $\eta_{p} /\left(d_{n} / \tau_{n}+1\right) \ll$ $p_{\varpi}^{\prime}(0+) /\left(d_{n} / \tau_{n}+1\right) \ll d_{n}$. Note that condition 3.2 requires $d_{n} / \tau_{n} \rightarrow \infty$ as shown in proof of proposition 4 so that $O\left(d_{n} / \tau_{n}+1\right)=O\left(d_{n} / \tau_{n}\right)$. Therefore, the valid range of $\tau_{n}$ is restricted as $\tau_{n}<d_{n}^{2} / \eta_{p}=o(1)$. 


\section{References}

Breheny, P. and Huang, J. (2011). Coordinate descent algorithms for nonconvex penalized regression, with applications to biological feature selection. The Annals of Applied Statistics, 5(1):232-253.

Fan, J. and Lv, J. (2011). Nonconcave penalized likelihood with np-dimensionality. Information Theory, IEEE Transactions on, 57(8):5467-5484.

McCullagh, P. and Nelder, J. (1989). Generalized linear models. Chapman \& Hall/CRC.

Zou, H. and Li, R. (2008). One-step sparse estimates in nonconcave penalized likelihood models. Annals of Statistics, 36(4):1509. 